\title{
Origin of the Quantum-Critical Transition in the Bilayer Heisenberg Model
}

\author{
C. N. A. van Duin and J. Zaanen \\ Institute Lorentz for Theoretical Physics, Leiden University, P.O.Box 9506, 2300 RA Leiden, The Netherlands
}

(Received 6 January 1997)

\begin{abstract}
The bilayer Heisenberg antiferromagnet is known to exhibit a quantum-critical transition at a particular value of the interlayer coupling. Using a new type of coherent state, appropriate to the special order parameter structure of the bilayer, we map the problem onto the quantum nonlinear sigma model. It is found that the bare coupling constant diverges at the classical transition, so that in any finite dimension the actual transition occurs inside the ordered phase of the classical theory. [S0031-9007(97)02986-4]
\end{abstract}

PACS numbers: 75.10.Jm, 64.60.-i, 71.27. +a, 75.45. $+\mathrm{j}$

The study of nonclassical collective quantum states of matter is a central theme of modern condensed matter physics. Despite the successes in $1+1$ dimensions, it has proven difficult to address these matters in higher dimensions. Either the minus-sign problem intervenes (as in, e.g., the $t-J$ model and frustrated spin models), or the tendency towards classical order is too strong (e.g., unfrustrated spin models). The class of bilayer Heisenberg models is special in this regard [1,2]. It is sign-free, and convincing numerical evidence exists showing that its longwavelength behavior is governed by the $O(3)$ quantum nonlinear sigma model (QNLS) with tunable bare coupling constant $u$ [3]. The relationship between the microscopic model and its long wavelength behavior is nontrivial. Chubukov and Morr (CM) made the key observation that, in order to construct the classical limit, the severe local (interplanar) fluctuations have to be integrated out first [4]. In the resulting singlet-triplet representation, a phase transition between a Néel state and an incompressible state is found already at the classical level. Here it is shown that this transition does not correspond to the quantum critical transition found in numerical studies. Because of the special structure of the order parameter, the standard SU(2) generalized spin coherent state does not suffice for the construction of the path integral. We introduce a novel type of coherent state which allows us to straightforwardly recover the QNLS describing the long wavelength behavior. We find that the bare coupling constant of the field theory diverges at the classical transition. The quantum phase transition therefore occurs well before the classical transition can occur, and the latter is therefore in any finite dimension an artifact. Our results are consistent with the indications of quantum criticality found by $\mathrm{CM}$.

It is convenient to consider the "bilayer" model in arbitrary dimensions, with an added magnetic field $(\vec{B})$,

$$
\begin{aligned}
\mathcal{H}= & J_{1} \sum_{\langle i j\rangle}\left(\vec{s}_{i 1} \cdot \vec{s}_{j 1}+\vec{s}_{i 2} \cdot \vec{s}_{j 2}\right) \\
& +J_{2} \sum_{i} \vec{s}_{i 1} \cdot \vec{s}_{i 2}-\vec{B} \cdot \sum_{i}\left(\vec{s}_{i 1}+\vec{s}_{i 2}\right),
\end{aligned}
$$

where $\langle i j\rangle$ runs over the bonds of two $d$-dimensional hypercubes 1 and 2 . The antiferromagnetically coupled
$\left(J_{1}>0\right) s=\frac{1}{2}$ Heisenberg spins $\vec{s}_{i \eta}$ are coupled locally by $J_{2}$. Following CM, we first integrate out the $J_{2}$ term $[4,5]$. Define the sum and the difference of the spin operators,

$$
\vec{S}=\vec{s}_{1}+\vec{s}_{2} ; \quad \overrightarrow{\tilde{S}}=\vec{s}_{1}-\vec{s}_{2},
$$

such that

$$
\begin{aligned}
\mathcal{H}= & \frac{1}{2} J_{1} \sum_{\langle i j\rangle}\left(\vec{S}_{i} \cdot \vec{S}_{j}+\overrightarrow{\tilde{S}}_{i} \cdot \overrightarrow{\tilde{S}}_{j}\right) \\
& +\frac{1}{4} J_{2} \sum_{i}\left(\vec{S}_{i}^{2}-\overrightarrow{\tilde{S}}_{i}^{2}\right)-\vec{B} \cdot \sum_{i} \vec{S}_{i} .
\end{aligned}
$$

Equation (2) amounts to a transformation to a singlettriplet basis. Introducing hard-core bosons creating the local singlet state, $a_{i}^{\dagger}=\frac{1}{\sqrt{2}}\left(c_{i 1 \downarrow}^{\dagger} c_{i 2 \uparrow}^{\dagger}-c_{i 1 \uparrow}^{\dagger} c_{i 2 \downarrow}^{\dagger}\right)$, and the local triplet $b_{i 1,0,-1}^{\dagger}\left(b_{1 i}^{\dagger}=c_{i 1 \uparrow}^{\dagger} c_{i 2 \uparrow}^{\dagger}\right.$ etc.), Eq. (2) can be alternatively written as

$$
\begin{aligned}
S^{z} & =b_{1}^{\dagger} b_{1}-b_{-1}^{\dagger} b_{-1}, \\
S^{+} & =\sqrt{2}\left(b_{1}^{\dagger} b_{0}+b_{0}^{\dagger} b_{-1}\right), \\
\tilde{S}^{z} & =-a^{\dagger} b_{0}-b_{0}^{\dagger} a, \\
\tilde{S}^{+} & =\sqrt{2}\left(b_{1}^{\dagger} a-a^{\dagger} b_{-1}\right) .
\end{aligned}
$$

$\vec{S}$ describes $S=1$ spins, while $\overrightarrow{\tilde{S}}$ is related to fluctuations from triplets to singlets. These operators form an $o(4)$ dynamical algebra,

$$
\begin{aligned}
& {\left[S^{a}, S^{b}\right]=\iota \varepsilon_{a b c} S^{c},} \\
& {\left[\tilde{S}^{a}, \tilde{S}^{b}\right]=\iota \varepsilon_{a b c} S^{c},} \\
& {\left[S^{a}, \tilde{S}^{b}\right]=\iota \varepsilon_{a b c} \tilde{S}^{c} .}
\end{aligned}
$$

At the $J_{2}=0$ point (two decoupled layers) the problem has an $O(4)$ global invariance, which is broken for any finite $J_{2}$, leaving only an invariance under the $\mathrm{SU}(2)$ subgroup Eq. (5). The unconventional aspect of this problem is that for positive $J_{2}$ the spontaneous symmetry breaking involves the generators $\tilde{S}$. The $J_{2}>0$ classical 
saddle-point of CM is easily seen to correspond to the vacuum amplitudes $(z=2 d)$,

$$
\begin{aligned}
\overrightarrow{\tilde{\Omega}}= & \frac{1}{N}\left\langle\sum_{i}(-1)^{i} \overrightarrow{\tilde{S}}_{i}\right\rangle=\sqrt{1-\frac{J_{2}^{2}}{J_{1}^{2} z^{2}}} \Theta\left(J_{1} z-J_{2}\right) \hat{n}, \\
n_{A}= & \frac{1}{N}\left\langle\sum_{i} a_{i}^{\dagger} a_{i}\right\rangle=\frac{1}{2}\left(1+\frac{J_{2}}{J_{1} z}\right) \Theta\left(J_{1} z-J_{2}\right) \\
& +\Theta\left(J_{2}-J_{1} z\right),
\end{aligned}
$$

where $\hat{n}$ is a vector on the unit sphere. The condensation of $\overrightarrow{\tilde{S}}$ [Eq. (8)] and the existence of a mean singlet density [Eq. (9)] is a direct ramification of the explicit symmetry breaking due to the interplanar coupling. $\quad \overrightarrow{\tilde{\Omega}}$ is still a vector order parameter, because $\overrightarrow{\tilde{S}}$ transforms as a vector under $\vec{S}$. It is therefore a Néel order state, albeit one with a variable local moment size, which implies that its long wavelength behavior should be described by the QNLS.

On the classical level it is found that $n_{A}$ is nonzero for all positive $J_{2}$, while $\overrightarrow{\tilde{\Omega}}$ vanishes continuously at $J_{2}=J_{1} z$, where $n_{A}$ becomes equal to one. This is the lowest order result found by CM. Regarding its formal status, it is easily seen that this classical theory becomes exact in infinite dimensions $[5,6]$. The energy fluctuations disappear in this limit: $\Delta E / E \propto 1 / \sqrt{N d}$. In addition, we note that $\overrightarrow{\tilde{\Omega}}$ also exists in $(2+1) \mathrm{D}$, at least in the vicinity of the quantum critical point: the correlation functions in terms of $\vec{s}_{1}$ and $\vec{s}_{2}$ go to zero at the transition with their ratios fixed according to Eq. (8) [3].

What is wrong with the assertion that this transition and the quantum-critical transition in $(2+1) \mathrm{D}$ are the same? The transition in infinite dimensions is a classical transition. In terms of the singlet-triplet basis, the quantum fluctuations disappear at the lattice cutoff and thermal fluctuations dominate at any finite temperature. The numerical study shows quantum criticality [3]: at zero temperature, the quantum fluctuations are scale independent. In the remainder we will show that this classical theory becomes pathological in the neighborhood of the classical transition.

Coherent state path integrals offer a convenient framework to study quantum order parameter fluctuations [7]. Because of the special status of the order parameter Eq. (8), the usual generalized spin coherent states do not suffice. Our key result is the discovery of a special coherent state for this type of order parameter structure. Next to the general requirements of normalizability and the existence of the identity, it should be demanded from coherent states that they reproduce all properties of the classical sector. Besides reproducing Eqs. (8) and (9), they should also allow for an $\vec{S}$ derived vacuum expectation value,

$$
\vec{\Omega}=\frac{1}{N}\left\langle\sum_{i} \vec{S}_{i}\right\rangle \text {. }
$$

We find that the following coherent state satisfies all these requirements:

$$
|\Omega \tilde{\Omega}\rangle=e^{\iota \phi S^{z}} e^{\iota \theta S^{y}} e^{\iota \theta_{2} S^{x}} e^{\iota \psi \tilde{S}^{y}}|\chi\rangle,
$$

with the reference state,

$$
|\chi\rangle=\left(\cos \chi a^{\dagger}+\sin \chi b_{0}^{\dagger}\right)|\mathrm{vac}\rangle \text {. }
$$

Equation (11) looks conventional. It refers to the various rotations related to the $O(4)$ symmetry. The novelty is Eq. (12): instead of the usual maximum weight state, this nonexact state underlies the order parameter structure Eqs. (8) and (9), with $\overrightarrow{\tilde{\Omega}}$ chosen along the $z$ axis, while $\chi$ is fixed by the explicit symmetry breaking interaction $\sim J_{2}$. The freedom implied by Eq. (11) might at first instance appear as redundant. However, it turns out that the stiffness in the temporal direction is caused entirely by the fluctuations from $\overrightarrow{\tilde{\Omega}}$ into the $\vec{\Omega}$ direction, and the four angles appearing in Eq. (12) take care of the independent rotations of $\overrightarrow{\tilde{\Omega}}$ and $\vec{\Omega}$. Explicitly, $\psi$ parametrizes a rotation from $\overrightarrow{\tilde{\Omega}}$ to $\vec{\Omega} \perp \overrightarrow{\tilde{\Omega}}(\vec{S} \cdot \overrightarrow{\tilde{S}}=0)$. The rotation of $\overrightarrow{\tilde{\Omega}}$ in the plane perpendicular to $\vec{\Omega}$ is parametrized by $\theta_{2}$. This is the only free rotation left to $\overrightarrow{\tilde{\Omega}}$ in a magnetic field. $\theta$ and $\phi$ fix the direction of $\vec{\Omega}$.

We obtain the following expressions for the vacuum amplitudes with respect to this coherent state

$$
n_{A}=\cos ^{2} \chi \cos ^{2} \psi,
$$

$\vec{\Omega}=\sin 2 \chi \sin \psi(-\cos \theta \cos \phi,-\cos \theta \sin \phi, \sin \theta)$,

$$
\overrightarrow{\tilde{\Omega}}=\sin 2 \chi \cos \psi\left[\cos \theta_{2} \hat{\theta}(\theta, \phi)-\sin \theta_{2} \hat{\phi}(\phi)\right],
$$

where $\hat{\theta}$ and $\hat{\phi}$ are the local unit vectors in the $\theta$ and $\phi$ directions, $\hat{\theta}=(\sin \theta \cos \phi, \sin \theta \sin \phi, \cos \theta)$ and $\hat{\phi}=(\sin \phi,-\cos \phi, 0)$. The identity becomes

$$
\begin{aligned}
1= & \int d \mu(\vec{\Omega}, \overrightarrow{\tilde{\Omega}})|\Omega \tilde{\Omega}\rangle\langle\Omega \tilde{\Omega}| \\
= & \frac{2}{\pi^{4}} \int_{0}^{\pi / 2} d \chi \int_{0}^{\pi / 2} d \psi \int_{0}^{2 \pi} d \theta_{2} \\
& \times \int_{0}^{2 \pi} d \phi \int_{-\pi / 2}^{\pi / 2} d \theta \cos \theta|\Omega \tilde{\Omega}\rangle\langle\Omega \tilde{\Omega}| .
\end{aligned}
$$

By taking expectation values with regard to $|\Omega \tilde{\Omega}\rangle$ (classical limit), we find the $O(3)$ invariant version of the mean-field theory of Chubukov and Morr. Minimization of the classical energy with regard to the coherent state angles yields

$$
\begin{gathered}
\cos 2 \chi_{0}=\frac{J_{2}}{J_{1} z}+\mathcal{O}\left(B^{2}\right), \\
\sin \psi_{0}=\frac{B}{J_{1} z} \sqrt{\frac{J_{1} z-J_{2}}{J_{1} z+J_{2}}}+\mathcal{O}\left(B^{2}\right),
\end{gathered}
$$

with $\theta$ and $\phi$ fixed such that $\vec{\Omega}$ points in the direction of the magnetic field. We recover the classical orderdisorder transition at $J_{2}=J_{1} z$, where both $\tilde{\Omega}$ and the induced magnetization $\Omega$ vanish according to Eqs. (14) and (15). 
The derivation of the path integral is standard [7]. Using the Trotter formula, the evolution operator in imaginary time is written as $\left(N_{t}\right.$ is the number of time slices, $\delta_{t}$ the imaginary time interval, $N_{t} \delta_{t}=\beta$ ),

$$
Z=\lim _{\substack{N_{t} \rightarrow \infty \\ \delta_{t} \rightarrow 0}} \operatorname{Tr}_{t} \prod_{l=1}^{N_{t}} e^{-\delta_{t} \sum_{i} \mathcal{H}_{i}} .
$$

Inserting the identity (16) at every intermediary time and expanding $Z$ to lowest order in $\delta_{t}$,

$$
\begin{aligned}
Z= & \lim _{\substack{N_{t} \rightarrow \infty \\
\delta_{t} \rightarrow 0}} \int \mathcal{D} \mu \prod_{l=1}^{N_{t}} \prod_{i}\left[\left\langle\Omega \tilde{\Omega}\left(t_{l}, i\right) \mid \Omega \tilde{\Omega}\left(t_{l+1}, i\right)\right\rangle\right. \\
& \left.-\delta_{t}\left\langle\Omega \tilde{\Omega}\left(t_{l}, i\right)\left|\mathcal{H}_{i}\right| \Omega \tilde{\Omega}\left(t_{l}, i\right)\right\rangle\right],
\end{aligned}
$$

where the integration measure $\mathcal{D} \mu$ is given by $\prod_{l=1}^{N_{t}} d \mu\left(\left\{\vec{\Omega}_{l}\right\},\left\{\overrightarrow{\tilde{\Omega}}_{l}\right\}\right)$, while $\left\{t_{l}\right\}$ is the set of intermediary times in the imaginary time interval $[0, \beta]$. The kinetic term in the action follows from the first term inside the square brackets,

$$
\prod_{i}\left\langle\Omega \tilde{\Omega}\left(t_{l}, i\right) \mid \Omega \tilde{\Omega}\left(t_{l+1}, i\right)\right\rangle=1+\iota \delta_{t} \Phi\left(t_{l}\right)+\mathcal{O}\left(\delta_{t}^{2}\right),
$$

with

$$
\begin{aligned}
\Phi & =\sum_{i} \sin 2 \chi_{i} \sin \psi_{i}\left(\sin \theta_{l} \partial_{t} \phi_{i}+\partial_{t} \theta_{2 i}\right) \\
& =-\sum_{i} \sin 2 \chi_{i} \vec{O}_{i} \cdot \partial_{t} \frac{\overrightarrow{\tilde{O}}_{i}}{\tilde{O}_{i}} \times \frac{\overrightarrow{\tilde{O}}_{i}}{\tilde{O}_{i}},
\end{aligned}
$$

where $\vec{O}=\vec{\Omega} / \sin 2 \chi$ and $\overrightarrow{\tilde{O}}=\overrightarrow{\tilde{\Omega}} / \sin 2 \chi$, so $O^{2}+$ $\tilde{O}^{2}=1$.

The potential energy is $(B=0)$,

$$
\begin{aligned}
\mathcal{V}= & \frac{J_{1}}{2} \sum_{\langle i, j\rangle} \sin 2 \chi_{i} \sin 2 \chi_{j}\left(\vec{O}_{i} \cdot \vec{O}_{j}+\overrightarrow{\tilde{O}}_{i} \cdot \overrightarrow{\tilde{O}}_{j}\right) \\
& +\frac{1}{4} J_{2} \sum_{i}\left(1-4 \tilde{O}_{i}^{2} \cos ^{2} \chi_{i}\right) .
\end{aligned}
$$

Taking the time continuum limit, the path integral becomes $Z=\int \mathcal{D} \mu e^{-S_{M}}$, with the real-time action

$$
S_{M}=\int_{0}^{T} d x_{0}\left[-\Phi\left(x_{0}\right)+\mathcal{V}\left(x_{0}\right)\right] .
$$

To derive the long wavelength theory, $\vec{O}$ and $\overrightarrow{\tilde{O}}$ are separated into a slowly varying order-parameter part and a rapidly fluctuating part which will be integrated out. The fluctuations in $\chi$ are massive because of the explicit symmetry breaking, and can be neglected. We are left with

$$
\begin{gathered}
\overrightarrow{\tilde{O}}_{i}=\eta_{i}\left(\overrightarrow{\tilde{m}}_{i}+a \overrightarrow{\tilde{L}}_{\| i}\right)+a \overrightarrow{\tilde{L}}_{\perp i}, \\
\vec{O}_{i}=\vec{m}_{i}+a \vec{L}_{i} .
\end{gathered}
$$

The (staggered) fluctuation $\overrightarrow{\tilde{L}}_{\| i}$ is parallel to the order parameter $\overrightarrow{\tilde{m}}_{i}\left(\eta_{i}= \pm 1\right.$ depending on the sublattice). $\vec{L}$ has a component along $\vec{m}$, but is perpendicular to $\overrightarrow{\tilde{m}}$ because of the constraint $\vec{O}_{i} \cdot \overrightarrow{\tilde{O}}_{i}=0$. As we already indicated, despite the fact that the order-parameter part of $\vec{O}$ is zero in the absence of a magnetic field, the fluctuations in this quantity are actually producing the stiffness in the time direction and should be carefully integrated out. We expand to second order in the lattice constant $a$, which will be taken to zero at the end of the calculation. Using the constraint $O_{i}^{2}+\tilde{O}_{i}^{2}=1$, the fluctuation $\overrightarrow{\tilde{L}}_{\| i}$ is eliminated from the action. Different from the single-layer system, two canting fields result, $\overrightarrow{\tilde{L}}_{\perp i}$ and $\vec{L}_{i}$, which have to be integrated out. The former does not influence the long wavelength behavior, while the latter is responsible for the kinetic term in the effective action.

After expanding in $a$ and eliminating $\overrightarrow{\tilde{L}}_{\|}$, the kinetic term becomes

$$
\Phi=-\sum_{i} \sin 2 \chi_{i} \frac{a}{\tilde{m}_{i}^{2}} \vec{L}_{i} \cdot \partial_{0} \overrightarrow{\tilde{m}}_{i} \times \overrightarrow{\tilde{m}}_{i}+\text { stagg. terms }
$$

Using $\overrightarrow{\tilde{m}}_{i}-\overrightarrow{\tilde{m}}_{j} \simeq a \partial_{i \rightarrow j} \overrightarrow{\tilde{m}}_{i}$, it can be seen that the staggered terms give contributions which are of third order in $a$. The expression for $\Phi$ is identical to that for the single-layer system, apart from the factor $\sin 2 \chi$ and the absence of a topological term. Within the limitations of the semiclassical expansion, the above derivation is in principle valid for any dimension, including the $1+1$ dimensional two leg spin ladder systems. The usual argument for the irrelevance of topological terms in these systems are based on the proximity of Néel order on both chains separately: the topological terms in the two rows cancel each other. Here we find that this holds regardless of the strength of the local fluctuations. We notice that, according to Haldane's conjecture [8], the spectrum of the two leg ladder has to be gapped for any $J_{2} \neq 0$.

The potential term is written in the form

$$
\begin{aligned}
\mathcal{V}= & J_{1} \sum_{\langle i, j\rangle} \sin 2 \chi_{i} \sin 2 \chi_{j} \\
& \times\left[\frac{a^{2}}{4}\left(\partial_{i \rightarrow j} \overrightarrow{\tilde{m}}_{i}\right)^{2}+a^{2}\left(L_{i}^{2}+\tilde{L}_{\perp i}^{2}\right)-2\right] \\
& +J_{2} \sum_{i} a^{2} L_{i}^{2} \cos ^{2} \chi_{i}-\frac{J_{2}}{4} \sum_{i}\left(1-4 \cos ^{2} \chi_{i}\right) .
\end{aligned}
$$

In the continuum limit $(a \rightarrow 0)$, the summations over sites are replaced by integrations over space, $\sum_{i} \rightarrow a^{-d} \int d^{d} x$. The $\mathcal{O}(1)$ term in Eq. (28), corresponding with the meanfield energy for the bilayer model, acquires a large prefactor $a^{-d}$ and can be integrated by steepest descent. This yields the mean-field expression for $\chi$, Eq. (17).

After integrating over the fluctuations $\vec{L}$ and $\overrightarrow{\tilde{L}}_{\perp}$ we recover the effective action responsible for the long wavelength fluctuations, which is the $O(3)$ QNLS,

$$
S_{M}=\frac{1}{2} \int d^{d+1} x\left[\chi_{\perp}\left(\partial_{0} \overrightarrow{\tilde{m}}\right)^{2}-\rho_{s} \sum_{\alpha=1}^{d}\left(\partial_{\alpha} \overrightarrow{\tilde{m}}\right)^{2}\right] .
$$


Although the form of Eq. (29) is dictated by symmetry, the parameters appearing in the effective theory have a quite different meaning in terms of the microscopic model than is the case in single layer problems. Taking the saddle-point values, the perpendicular susceptibility and the spin stiffness become, respectively,

$$
\begin{gathered}
\chi_{\perp}=a^{-d} \frac{J_{1} z-J_{2}}{J_{1}^{2} z^{2}}, \\
\rho_{s}=a^{2-d} \frac{J_{1}}{2}\left(1-\frac{J_{2}^{2}}{J_{1}^{2} z^{2}}\right) .
\end{gathered}
$$

Both the susceptibility and the spin stiffness vanish at the classical transition at $J_{2}=z J_{1}$. The spin-wave velocity $v_{s}=\sqrt{\rho_{s} / \chi_{\perp}}$ remains finite at the transition, and no divergencies occur on the Gaussian level [4]. The stability of the classical state against quantum melting is, however, controlled by the dimensionless coupling constant $u=a^{1-d} / \sqrt{\rho_{s} \chi_{\perp}}$, which is found to diverge at the classical transition as $u \sim 1 / J_{2}^{*}$, where $J_{2}^{*}$ is the reduced interlayer coupling $J_{2}^{*}=\left(J_{1} z-J_{2}\right) / J_{2}$. In any finite dimension, the $O(3)$ QNLS quantum critical transition occurs at a finite value of the coupling constant and it follows that the long wavelength fluctuations destroy the $\tilde{\Omega}$ type Néel order before the classical critical point is reached. Accordingly, the quantum critical transition of the bilayer model is of the $O(3)$ QNLS kind, and the classical transition exists only in infinite dimensions.

This theory is even quantitatively reasonable. One loop renormalization theory for the QNLS in $2+1$ dimensions puts the critical coupling at $u^{*}=4 \pi$ [9]. Using the saddle-point values for the spin stiffness and susceptibility [Eq. (31)], we find the quantum transition to occur at $J_{2}^{c} / J_{1}=3.3$. Given that $1 / S$-like corrections are neglected [5], the agreement with the value of 2.5-2.6 obtained from quantum Monte Carlo [3] and series expansions [2] is reasonable.

In summary, we have clarified the origin of the quantum critical transition of the bilayer Heisenberg problem. The key aspect is that the order parameter structure as discovered by Chubukov and Morr [4] is unusual. Although this order parameter is macroscopically of the usual $O(3)$ vector kind, and therefore described by the $O(3)$ quantum nonlinear sigma model, its microscopic status is unconventional. The operators acquiring a vacuum amplitude $(\tilde{S})$ are not the ones expressing the global SU(2) invari- ance of the problem. This kind of order parameter structure arises naturally in the present context and we expect it to be quite common in the general context of quantum magnetism [10]. Our main result is the discovery of a new type of spin coherent state which allows for the requantization of such order parameter structures. As applied to the bilayer problem, the novelty is that in any finite dimension the classical theory becomes highly pathological: the bare coupling constant of the field theory diverges at the classical transition, explaining why the quantum transition obeys $O(3)$ QNLS universality.

We acknowledge very helpful discussions with A. Chubukov and S. Sachdev. Financial support was provided by the Foundation of Fundamental Research on Matter (FOM), which is sponsored by the Netherlands Organization for the Advancement of Pure Research (NWO), and by the Dutch Academy of Sciences (KNAW).

[1] T. Matsuda and K. Hida, J. Phys. Soc. Jpn. 59, 2223 (1990); A. J. Millis and H. Monien, Phys. Rev. Lett. 70, 2810 (1993); Phys. Rev. B 50, 167606 (1994).

[2] K. Hida, J. Phys. Soc. Jpn. 61, 1013 (1992).

[3] A. W. Sandvik and D. J. Scalapino, Phys. Rev. Lett. 72, 2777 (1994).

[4] A. V. Chubukov and D. K. Morr, Phys. Rev. B 52, 3521 (1995).

[5] C. N. A. van Duin and J. Zaanen (to be published).

[6] The same holds for infinite ranged intraplanar interactions: C. Gros, W. Wenzel, and J. Richter, Europhys. Lett. 32, 747 (1995). It is noted that the spectrum of physical excitations is in this case gapped. The mode-softening transition discussed by Gros et al. occurs actually in the thermodynamically irrelevant thin spectrum. E. Lieb and D. Mattis, J. Math. Phys. 3, 749 (1962); T. A. Kaplan, W. von der Linden, and P. Horsch, Phys. Rev. B 42, 4663 (1990).

[7] E. Fradkin, Field Theories of Condensed Matter Systems (Addison-Wesley, Reading, MA, 1991); A. Auerbach, Interacting Electrons and Quantum Magnetism (SpringerVerlag, New York, 1994).

[8] F. D. M. Haldane, Phys. Rev. Lett. 50, 1153 (1983).

[9] S. Chakravarty, B.I. Halperin, and D. R. Nelson, Phys. Rev. Lett. 60, 1057 (1988); Phys. Rev. B 39, 2344 (1989); A. V. Chubukov, S. Sachdev, and J. Ye, Phys. Rev. B 49, 11919 (1994).

[10] For instance, the so-called singlet-triplet models: P. Fulde and I. Peschel, Adv. Phys. 21, 1 (1972). 